\title{
The Utility of the Mini-Addenbrooke's Cognitive Examination as a Screen for Cognitive Impairment in Elderly Patients with Chronic Kidney Disease and Diabetes
}

\author{
Peter Hobson $^{a} \quad$ Kamel H. Rohoma ${ }^{a, b}$ Stephen P. Wong ${ }^{a}$ \\ Mick J. Kumwenda ${ }^{a}$ \\ a Glan Clwyd Hospital, Betsi Cadwaladr University Health Board, NHS Wales, \\ Bodelwyddan, UK; ${ }^{b}$ Internal Medicine Department, Faculty of Medicine, Alexandria \\ University, Alexandria, Egypt
}

\author{
Keywords \\ Chronic kidney disease - Diabetes - Screening for cognitive impairment - Mild cognitive \\ impairment · Mini-Addenbrooke's Cognitive Examination
}

\begin{abstract}
Background/Aims: We tested the utility of the Mini-Addenbrooke's Cognitive Examination (M-ACE) in a cohort of older adults with chronic kidney disease (CKD) and diabetes. Method: The M-ACE was administered to 112 CKD and diabetes patients attending a nephrology clinic. Cognitive impairment was based upon patient, informant, and case review, neuropsychological assessment, and application of criteria for mild cognitive impairment (MCI) and the Diagnostic and Statistical Manual of Mental Disorders, fifth edition for dementia. The M-ACE was also compared to the Mini-Mental State Examination (MMSE). Results: Upon assessment, 52 patients had normal cognitive function, 33 had $\mathrm{MCI}$, and 27 had dementia. The area under the receiver operating curve for the M-ACE was 0.96 (95\% CI 0.95-1.00). The sensitivity and specificity for a dementia diagnosis were 0.96 and 0.84 at the cut point $<25$ and 0.70 and 1.00 at the cut point $<21$. Mean M-ACE scores differed significantly between normal, demented, and $\mathrm{MCI}$ groups $(p<0.001)$, and compared to the MMSE, the M-ACE did not suffer from ceiling effects. Conclusion: The M-ACE is an easily administered test with good sensitivity and specificity to capture and assist in the diagnosis of MCI or dementia in patients with CKD and diabetes.


Hobson et al.: M-ACE as a Cognitive Screen in Diabetic and Kidney Disease Patients

\section{Introduction}

As populations age throughout the world, inevitably there will be a rise in illnesses associated with ageing, such as chronic kidney disease (CKD), diabetes, as well as cerebrovascular and neurological disorders [1-4]. Although the prevalence of dementia and its associated social and economic costs will rise exponentially with the ageing population, more recent general population investigations have reported declining incidence rates [5-7]. This may in part be due to potentially earlier and more appropriate primary and secondary care interventions, targeted at population disorders, such as cardiovascular health. This would suggest that screening of at-risk populations is warranted.

Cognitive impairment is a frequently reported comorbidity in advanced- and end-stage CKD patients [8-11]. However, recent investigations have reported that the early and more moderate stages of CKD are also independent risk factors for the development of cognitive impairment, with reported prevalence rates ranging from $21 \%$ to over $60 \%$, depending on the stage of the disease and the methodology employed [12-14]. It has also been estimated that compared to the general population, CKD patients have a 2 -fold increased risk for the development of dementia [15]. Chronic diseases, such as CKD and diabetes, and their associated vascular risks may benefit from early intervention and management to delay or reduce the risk of developing cognitive impairment or dementia. It is then important to determine if existing cognitive assessments are reliable and valid measures in CKD populations who are at increased risk for dementia. However, recognising, diagnosing, and managing cognitive impairment, especially in busy clinical settings, is often challenging because of limited knowledge or reluctance to confidently use cognitive assessments. A brief global screening instrument that does not require specialist training, apparatus, or interpretation to complete is needed, especially in these settings.

The aim of this investigation was to determine the utility of the recently introduced shortened version of the Addenbrooke's Cognitive Examination (ACE), the 30-item mini-ACE (M-ACE), as a cognitive screen in a cohort of older adults with CKD and diabetes [16].

\section{Subjects and Methods}

A community sample of 118 outpatient clinic attendees aged 60 years and older with an established diagnosis of CKD with an estimated glomerular filtration rate (eGFR based on MDRD equation) of $<60 \mathrm{~mL} / \mathrm{min} / 1.73 \mathrm{~m}^{2}$, non-dialysis dependent, with diabetes and without a known preexisting diagnosis of a stroke, cognitive impairment, or dementia, were invited to participate in the investigation.

All patients were screened with the ACE III and the Mini-Mental State Examination (MMSE) $[17,18]$. The M-ACE scores were obtained from the more comprehensive ACE-III. The M-ACE assesses the cognitive domains of attention (4 points), letter fluency (7 points), the clock drawing test ( 5 points), and memory learning and recall ( 7 points each, with a maximum of 14 points), where cut points of 25 and 21 are sensitive to cognitive impairment (see online suppl. file for a copy of the M-ACE; www.karger.com/doi/10.1159/000450784).

The diagnosis of cognitive impairment was based upon patient and informant review, clinical case review, neuropsychological assessment, and application of DSM-V criteria for dementia [19]. The diagnosis of mild cognitive impairment (MCI), was based upon patient, caregivers', informants', or clinicians' observed reports of symptoms of memory impairment, decline in the ability to perform everyday activities (though still able to perform these activities without assistance), and difficulties with language, perceptual-motor, and social skills. These impairments were in the absence of delirium or dementia. In addition, the MCI diag- 
nosis will be based upon objective evidence of impairment in one or more areas of cognition (e.g., memory, attention, language, visuospatial skills, and executive function) and impairments 1-2 standard deviations (SD) below population norms and application of DSM-V criteria $[20,21]$.

\section{Ethics}

All patients gave written and informed consent and approval was given by the local research and ethics committee.

\section{Statistical Analysis}

The demographic characteristics of the patients, as well as their baseline neuropsychological assessments, were summarised with descriptive statistics including mean, SD, median, and standard error of the mean (SE). In addition, the strength of linear association between variables was calculated with Spearman correlation coefficients. Visual examination and analysis of the M-ACE distribution with Shapiro-Wilk test revealed that it was non-normal $(p<0.001)$; consequently, nonparametric Mann-Whitney $\mathrm{U}$ analysis was used for betweengroup comparisons.

Receiver operating characteristic (ROC) analysis was employed to determine the proportion of patients correctly classified as having MCI or dementia. Positive likelihood ratio $(\mathrm{LR}+)$ was calculated: the ratio between the probability of a positive test result given the presence of MCI or dementia and the probability of a positive test result given the absence of either MCI or dementia was calculated, i.e., true positive rate/false positive rate = sensitivity/ (1 - specificity). The effect size was calculated with Cohen's $d$ (the difference between 2 means divided by SD for the data) for the diagnosis of MCI and dementia [22]. Cohen's $d$ effect sizes $>0.8$ are considered to be large. Predictive variables for the development of either MCI or dementia were explored with backward Wald likelihood ratio (LR) logistic regression analysis.

All analysis was performed using SPSS V22 software, except for Cohen's $d$. The level of significance was set at $p<0.05$.

\section{Results}

Upon assessment, 6 patients were unable to complete the ACE: 4 due to significant visual field loss, 1 due to significant premorbid learning difficulties, and 1 patient refused to participate, leaving a cohort of 112 patients. The cohort $(n=112)$ comprised 65 male and 47 female patients aged between 62 and 93 years (mean 77.1, SD 7.9). The demographic, clinical details (CKD and diabetes, eGFR, $\mathrm{HbA}_{1 \mathrm{c}}, \mathrm{Hb}$, and albumin/creatinine ratio results) and assessments of the patients are given in Table 1.

The overall mean M-ACE and MMSE scores were 23.3 (SD 4.78; SE 0.51; 95\% CI 22.224.4) and 25.1 (SD 4.4; SE 0.49; 95\% CI 24.1-26.1), respectively. In total, 27 patients fulfilled DSM-IV criteria for dementia and further 33 fulfilled criteria for MCI. The total M-ACE scores differed significantly between normal, demented, and MCI groups $(p<0.001)$. Comparing the individual cognitive domains of the M-ACE between the cognitively normal and the demented patients revealed that demented patients had worse cognitive function in all but the attention domain (Table 2). This analysis was repeated with the MCI patients and revealed significant differences in the cognitive domains of fluency and memory (Table 2).

Although the item content of the M-ACE and the MMSE only overlaps in the domain of orientation in the demented patients, there was a moderate correlation between the assessments $\left(r_{s}=0.64, p<0.001\right)$ indicating good criterion validity. The internal reliability of the 
Table 1. Demographic and clinical indices of the normal, MCI, and demented groups

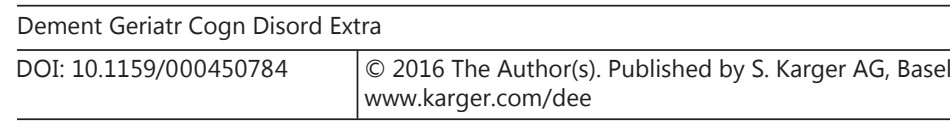

Hobson et al.: M-ACE as a Cognitive Screen in Diabetic and Kidney Disease Patients

\begin{tabular}{lccc}
\hline & $\begin{array}{l}\text { NCI } \\
(n=52)\end{array}$ & $\begin{array}{l}\text { MCI } \\
(n=33)\end{array}$ & $\begin{array}{l}\text { Demented } \\
(n=27)\end{array}$ \\
\hline Age, years & $76.4(7.4)$ & $78.1(10.1)$ & $79.8(5.4)$ \\
Gender (M:F) & $27: 13$ & $18: 12$ & $16: 10$ \\
Education, years & $10.9(1.9)$ & $10.7(1.8)$ & $10.5(2.5)$ \\
eGFR, ml/min & $29.9(9.6)$ & $29.7(7.9)$ & $31.9(11.7)$ \\
$\quad$ CKD stage 3a & $47.8(2.4)$ & $47.2(5.1)$ & $49.7(5.6)$ \\
$\quad$ CKD stage 3b & $34.9(3.8)$ & $34.2(4.2)$ & $34.5(5.2)$ \\
$\quad$ CKD stage $\geq 4$ & $22.2(4.9)$ & $25.8(2.8)$ & $24.7(3.1)$ \\
Creatinine, $\mu$ mol/L & 175.5 & 161.5 & 170.1 \\
HbA 1 , mmol/mol & $57.9(12.9)$ & $63.3(16.5)$ & $54.8(10.6)$ \\
Hb, g/L & $121.1(11.5)$ & $120.1(13.3)$ & $121.5(14.8)$ \\
Albumin & $34.8(4.5)$ & $34.8(4.2)$ & $34.4(3.3)$ \\
ACR & 4.8 & 5.8 & 4.6 \\
MMSE & $29.0(0.85)^{\dagger}$ & $26.3(1.7)^{\dagger}$ & $22.7(3.4)^{\dagger}$ \\
ACE III total & $92.1(4.9)^{\dagger}$ & $82.9(4.4)^{\dagger}$ & $66.4(15.6)^{\dagger}$ \\
\hline
\end{tabular}

All data reported are mean and SD, except for gender (proportions), ACR, and creatinine (median values). NCI, no cognitive impairment; ACR, albumin/creatinine ratio. ${ }^{\dagger}$ Significant differences between the normal, MCI, and demented patients, $p<0.001$.
Table 2. Cognitive domain comparisons of the M-ACE between the normal, MCI, and demented patients

\begin{tabular}{lccc}
\hline & NCI & MCI & Demented \\
\hline Attention & $3.9(0.30)$ & $3.9(0.25)$ & $3.6(0.81)$ \\
Fluency & $5.6(0.99)^{\dagger}$ & $4.8(1.16)^{*}$ & $3.1(0.81)$ \\
Visuospatial & $4.7(0.53)^{\dagger}$ & $4.4(0.93)$ & $3.1(1.58)$ \\
Memory & $12.6(1.63)^{\dagger}$ & $9.5(1.95)^{*}$ & $7.8(3.40)$ \\
M-ACE total & $27.1(1.95)^{\dagger}$ & $22.5(1.91)^{*}$ & $17.2(4.5)$ \\
\hline
\end{tabular}

The data reported are means and SD. NCI, no cognitive impairment. † Significant differences between the normal and demented patients, $p<0.001$. * Significant differences between normal and MCI patients, $p<0.001$.

M-ACE was further assessed with Cronbach's alpha statistic which found good internal consistency of 0.81 , which is in excess of the 0.7 standard [23]. Age, educational attainment, mood, stage of CKD (eGFR), and $\mathrm{HbA}_{1 \mathrm{c}}$ outcomes were not found to be significantly associated with the global M-ACE scores $(p>0.05)$.

The sensitivity and specificity of the M-ACE to detected dementia at the recommended cut point of $\leq 25$ were 96.5 and 84.3 (95\% CI 87.9-99.6 and 71.4-93), with a LR+ of 6.2 and a positive predictive value (PPV) of 87.3 . At the cut point of $\leq 21$, the sensitivity and specificity were 0.70 and 1.00 (95\% CI 0.51-0.85 and 0.95-1.00), with a LR+ of 100 (95\% CI 3.3-11.7) and a PPV of 100 . The LR+ for the M-ACE at $\geq 25 / 30$ indicates that patients who score within this range are 6 times more likely to have a dementia diagnosis and at $\geq 21 / 30$, specificity of 1.0 indicates a very high probability of a dementia diagnosis. The area under the receiver operating curve (AUC) for the M-ACE was 0.96 (SE 0.13; 95\% CI 0.95-1.00) and 0.95 (SE 0.23; 95\% CI 0.90-0.99) for the MMSE (Fig. 1). The M-ACE was weaker at detecting MCI at the cut 
Fig. 1. ROC curves of the M-ACE and MMSE sensitivity and specificity to detect cognitive impairment.

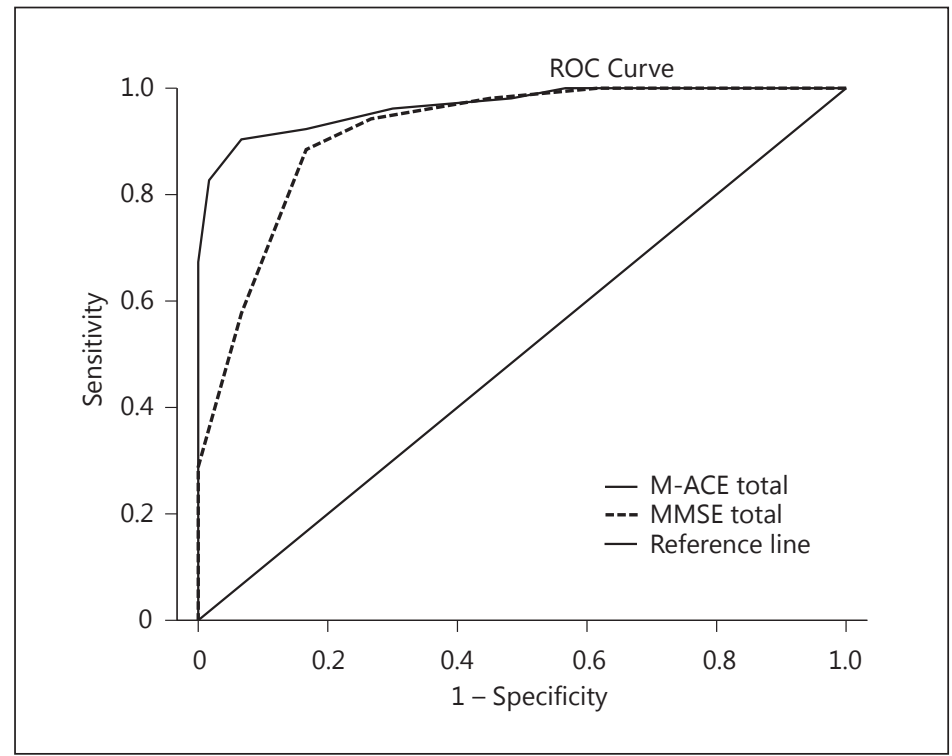

point of 25, with a sensitivity and specificity of 0.9 and 0.53 (95\% CI $0.74-0.98$ and $0.41-$ 0.64 ), with a LR+ of 1.9 and a PPV of 0.43 . There were no significant AUC differences between the M-ACE and MMSE $(p>0.05)$.

The effect sizes (Cohen's $d$ ) of the M-ACE were large for the diagnosis of dementia and MCI, with values of 2.8 and 2.4, respectively. Similarly, the MMSE had large effect sizes of 2.3 and 1.7 for dementia and $\mathrm{MCI}$, respectively.

Logistic regression analysis of the cognitive domains of the MCI patients revealed that memory recall was a significant predictive variable, with an odds of 1.96 (95\% CI 1.43-2.57), Wald $\chi^{2}$ (df 1) 18.94, $p<0.0001$, explaining $48 \%$ of the variance (Nagelkerke $R^{2}$ ). This suggests that in this cohort, neuropsychological impairment in this cognitive domain is significantly predictive of a single-domain amnesic MCI subtype. The analysis was repeated with the dementia patients and the significant predictive variables revealed were as follows: memory recall, odds 1.812 (95\% CI 1.21-2.70), Wald $\chi^{2}$ (df 1) 8.49, $p<0.004$; fluency, odds 3.419 (95\% CI 1.68-6.95), Wald $\chi^{2}$ (df 1) 11.54, $p<0.001$; and visuospatial (clock drawing test), odds 4.79 (95\% CI 2.00-11.41), Wald $\chi^{2}$ (df 1) 12.49, $p<0.0001$. Around $75 \%$ of the variance $\left(R^{2}\right)$ was explained by impairments in these cognitive domains.

\section{Discussion}

This investigation of CKD patients with diabetes found that the M-ACE cognitive screen had acceptable face, content, criterion, and construct validity. The sensitivity and specificity of the assessment to detect dementia at the recommended specified cut points of 25/30 and $21 / 30$ were found to be excellent. Patients whose scores fell at or below the recommended cut point of $25 / 30$ on the M-ACE were 6 times more likely to be demented than those without cognitive impairment. The high specificity observed at the lower M-ACE cut point of 21/30 suggests that those scoring within this range were almost definitely likely to fulfil criteria for dementia. In the current investigation, the M-ACE was not found to be vulnerable to the effects of age, educational attainment, CKD stage, or diabetes severity. 
Fig. 2. Error bar summarizing the distribution of the total M-ACE score by the DSM-V outcome. The circle in the middle of the bars represents the mean score and the whiskers are the $95 \%$ confidence intervals. NCI, no cognitive impairment.

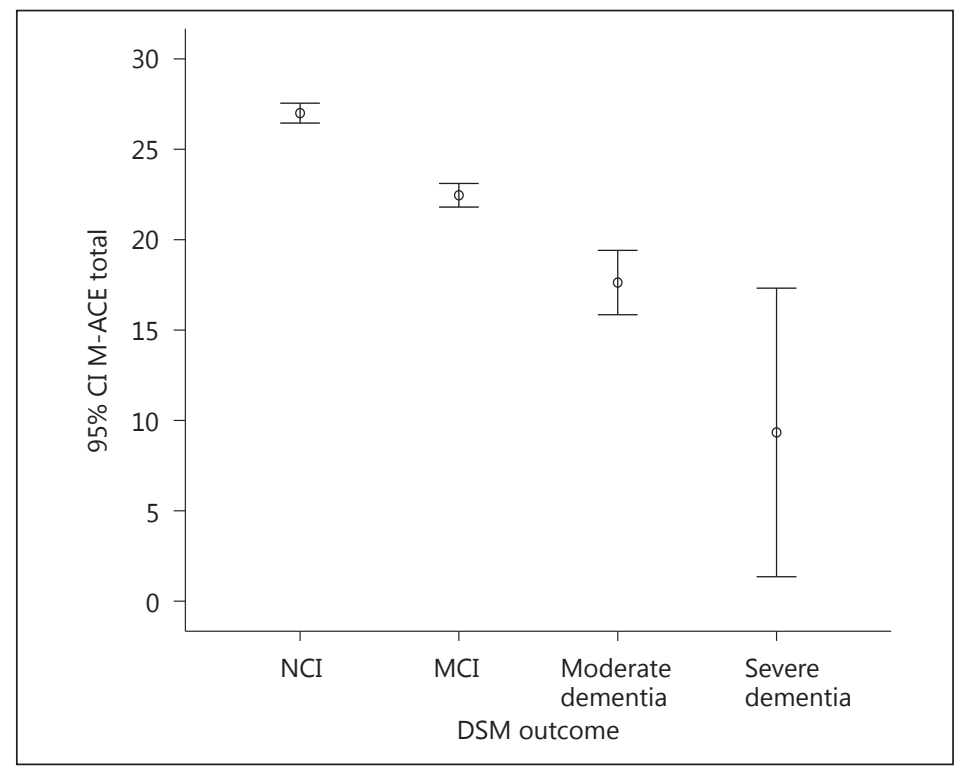

An examination of the item domain scores of the M-ACE revealed that the measure was able to distinguish between patients with and those without varying cognitive impairments (Fig. 2). The M-ACE is sensitive to detect varying degrees of cognitive impairment and does not suffer from ceiling effects, which is often observed in other brief global measures of cognitive function. It is also easily administered in a busy clinic and on average takes less than 5 min to administer. Additionally, compared to more formal neuropsychological tests, the M-ACE does not require highly trained professionals to administer or interpret its results. However, longitudinal investigations in this and other populations are needed to establish the M-ACE's test re-test reliability, interobserver reliability and sensitivity to change, and ability to differentiate between $\mathrm{MCI}$ subtypes and differing clinical cohorts.

Comparing the present study with previous M-ACE investigations needs to be interpreted cautiously because most have drawn their cohorts from specialist clinical research populations, patients who have been referred for assessment because of the subjective memory or cognitive complaints, or from memory clinics where patients have an established dementia diagnosis $[17,24]$. However, despite these methodological differences, our investigation reported similar levels of diagnostic sensitivity and specificity for MCI and dementia. Furthermore, the absence of ceiling effects with the M-ACE suggests that it will be a valuable tool for repeated assessments, particularly in patients with $\mathrm{MCI}$ or suspicion of dementia. We would suggest that the degree of cognitive impairments is more informative rather than a diagnostic label of dementia. This is particularly important since a diagnosis of dementia often impinges not only on the patients' medical treatments, investigations, and prognosis, but also their legal rights to, for example, drive a car or manage their own financial affairs.

This is the first investigation to our knowledge to assess the utility of the M-ACE in a typical CKD and diabetes outpatient clinic population. Whilst none of the patients in our investigation had a known diagnosis of cognitive impairment or dementia before the assessment, we did find a significant number of new cases of MCI and dementia.

This would suggest that the burden of cognitive impairments in CKD patients with diabetes may be underestimated $[25,26]$. In addition to this, a recent pooled analysis of 741 studies worldwide on the prevalence of diabetes suggests that since 1980 it has increased 4 -fold [27]. It was reported that nearly $40 \%$ of this increase was due to population growth 
and ageing. In view of the strong association of diabetes with ageing, vascular disease, and CKD, it is likely that a significant proportion of these patients are at risk of developing cognitive impairment. It could be argued that failing to adequately assess cognitive problems may reduce the benefit of an intervention programme or the effectiveness of complex medication regimes, or may explain patient noncompliance of pharmacological treatments and advice. This is of particular importance in advanced- and end-stage CKD patients who are expected to make important decisions about their future social, medical care and options for treatment interventions. Earlier recognition of cognitive impairment could also improve access to health and social care resources and possibly improve healthcare intervention outcomes.

We would caution the use of the M-ACE in isolation, or indeed any brief global neuropsychological assessment as a diagnostic tool. The main purpose of the brief instruments, such as the M-ACE, is to screen individuals quickly, with reasonable accuracy, in whom there is an increased risk of developing MCI or dementia. Patients who are found to be at risk will need more comprehensive assessments to reach a formal diagnosis.

In conclusion, the M-ACE is able to detect cognitive impairments at an early stage and could be employed to screen patients in the primary and secondary care settings. Additionally, the M-ACE, compared to the MMSE, is copyright free for clinical use and, unlike most brief global assessments, does not require specific test materials, just the (examiner's) recall of the test items and a blank sheet of paper to administer the clock drawing task and to record the test results.

\section{Acknowledgements}

We would like to show our appreciation to the patients and their families who assisted us in this research investigation.

This investigation was supported by funding from Betsi Cadwaladr University Health Board.

\section{Disclosure Statement}

The authors in this investigation do not have a have any disclosures.

\section{References}

1 Matthews FE, Arthur A, Barnes LE, et al: A two-decade comparison of prevalence of dementia in individuals aged 65 years and older from three geographical areas of England: results of the Cognitive Function and Ageing Study I and II. Lancet 2013;382:1405-1412.

2 Rocca WA, Petersen RC, Knopman DS, et al: Trends in the incidence and prevalence of Alzheimer's disease, dementia, and cognitive impairment in the United States. Alzheimers Dement 2011;7:80-93.

-3 Parikh NI, Hwang S-J, Larson MG, et al: Chronic kidney disease as a predictor of cardiovascular disease (from the Framingham Heart Study). Am J Cardiol 2008;102:47-53.

4 Dorsey ER, Constantinescu JP, Thompson KM, et al: Projected number of people with Parkinson's disease in the most populous nations, 2005 through 2030. Neurology 2007;68:384-386.

$\checkmark 5$ Satizabal CL, Beiser AS, Chouraki V, et al: Incidence of Dementia over Three Decades in the Framingham Heart Study. N Engl J Med 2016;374:523-532.

-6 Schrijvers EM, Verhaaren BF, Koudstaal PJ, Hofman A, Ikram MA, Breteler MM: Is dementia incidence declining? Trends in dementia incidence since 1990 in the Rotterdam Study. Neurology 2012;78:1456-1463.

7 Qiu C, von Strauss E, Bäckman L, Winblad B, Fratiglioni L: Twenty-year changes in dementia occurrence suggest decreasing incidence in central Stockholm, Sweden. Neurology 2013;80:1888-1894.

-8 Sehgal AR, Grey SF, DeOreo PB, et al: Prevalence, recognition, and implications of mental impairment among haemodialysis patients. Am J Kidney Dis 1997;30:41-49. 
-9 Murray AM, Tupper DE, Knopman DS, et al: Cognitive impairment in hemodialysis patients is common. Neurology 2006;67:216-223.

10 Griva K, Stygall J, Hankins M, et al: Cognitive impairment and 7-year mortality in dialysis patients. Am J Kidney Dis 2010;56:693-703.

11 Palmer SC, Ruospo M, Barulli MR, et al: COGNITIVE-HD study: protocol of an observational study of neurocognitive functioning and association with clinical outcomes in adults with end-stage kidney disease treated with haemodialysis. BMJ Open 2015;5:e009328.

12 Kurella M, Chertow GM, Luan J, Yaffe K: Cognitive impairment in chronic kidney disease. J Am Geriatr Soc 2004;52:1863-1869.

13 Khatri M, Nickolas T, Moon YP, et al: CKD associates with cognitive decline. J Am Soc Nephrol 2009;20:24272432.

14 Murray AM, Knopman DS: Cognitive impairment in CKD: no longer an occult burden. Am J Kidney Dis 2010: 56:615-618.

-15 Etgen T, Sander D, Chonchol M, et al: Chronic kidney disease is associated with incident cognitive impairment in the elderly: the INVADE study. Nephrol Dial Transplant 2009;24:3144-3150.

-16 Hsieh S, McGrory S, Leslie F, Dawson K, et al: The Mini-Addenbrooke's Cognitive Examination: a new assessment tool for dementia. Dement Geriatr Cogn Disord 2015;39:1-11.

17 Hsieh S, Schubert S, Hoon C, et al: Validation of the Addenbrooke's Cognitive Examination III in frontotemporal dementia and Alzheimer's disease. Dement Geriatr Cogn Disord 2013;36:242-250.

18 Folstein MF, Folstein SE, McHugh PR: "Mini-mental state". A practical method for grading the cognitive state of patients for the clinician. J Psychiatr Res 1975;12:189-198.

19 American Psychiatric Association: Diagnostic and Statistical Manual of Mental Disorders, ed 5. Washington, 2013.

20 Petersen RC, Doody R, Kurz A, et al: Current concepts in mild cognitive impairment. Arch Neurol 2001;58: 1985-1992.

21 Petersen RC: Mild Cognitive Impairment. N Engl J Med 2011;364:2227-2234.

22 Cohen J: Statistical Power Analysis for the Behavioral Sciences, ed 2. Hillsdale, Lawrence Earlbaum Associates, 1998.

23 Nunnally JC: Psychometric Theory, ed 2. New York, McGraw-Hill, 1978.

-24 Larner AJ: Mini-Addenbrooke's Cognitive Examination: a pragmatic diagnostic accuracy study. Int J Geriatr Psychiatry 2015;30:547-548.

-25 Schneider SM, Kielstein JT, Braverman J, Novak M: Cognitive function in patients with chronic kidney disease: challenges in neuropsychological assessments. Semin Nephrol 2015;35:304-310.

26 Seidel UK, Gronewold J, Volsek M, Todica O, Kribben A, et al: The prevalence, severity, and association with HbA1c and fibrinogen of cognitive impairment in chronic kidney disease. Kidney Int 2014;85:639-702.

27 NCD Risk Factor Collaboration (NCD-RisC): Worldwide trends in diabetes since 1980: a pooled analysis of 751 population-based studies with 4.4 million participants. Lancet 2016;387:1513-1530. 